\title{
An Integrated, Robust Approach to Lane Marking Detection and Lane Tracking
}

\author{
Joel C. McCall and Mohan M. Trivedi \\ jmccall@ucsd.edu, mtrivedi@ucsd.edu \\ Computer Vision and Robotics Research Laboratory \\ University of California, San Diego \\ La Jolla, CA, 92093-0434 \\ http://cvrr.ucsd.edu
}

\begin{abstract}
Lane Detection is a difficult problem because of the varying road conditions that one can encounter while driving. In this paper we propose a method for lane detection using steerable filters. Steerable filters provide robustness to lighting changes and shadows and perform well in picking out both circular reflector road markings as well as painted line road markings. The filter results are then processed to eliminate outliers based on the expected road geometry and used to update a road and vehicular model along with data taken internally from the vehicle. Results are shown for a 9000-frame image sequence that include varying lane markings, lighting conditions, showing, and occlusion by other vehicles.
\end{abstract}

\section{Introduction}

Lane detection is a well-researched area of computer vision with applications in autonomous vehicles and driver support systems. This is in part because, despite the perceived simplicity of finding white markings on a dark road, it can be very difficult to determine lane markings on various types of road. These difficulties arise from shadows, occlusion by other vehicles, changes in the road surfaces itself, and differing types of lane markings. A lane detection system must be able to pick out all manner of markings from cluttered roadways and filter them to produce a reliable estimate of the vehicle position and trajectory relative to the lane as well as the parameters of the lane itself such as its curvature and width.

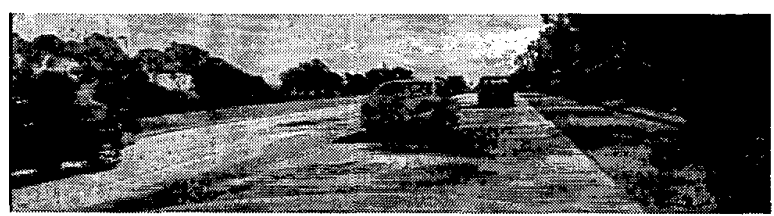

Figure 1 An example of a highway road, lane markings vary from bots dots to solid lines and are cluttered by shadows and other vehicles.
Figure 1 shows examples of the varying highway markings that must be looked at when creating a lane detector. The left hand lane markings on this are bots dots (circular reflectors) while a solid white line marks the right hand lane. Both lane markings show poor contrast. The trees on the side of the highway in Figure 1 cast shadows over the lane markers, further cluttering the image.

In this paper we propose a method for lane detection that can work on a variety of different road types under a variety of lighting changes and shadowing. In order to do this we use steerable filters [1] which can be convolved with the input image and provide features that allow them to be used to detect both bots dots and solid lines while providing robustness to cluttering and lighting changes.

\subsection{Previous Work}

Many researchers have shown lane detectors based on a wide variety of techniques. A technique commonly used is based on detecting edges and fitting lines to these edges via the Hough transform [2]. The Hough transform is often sensitive to clutter from shadows and varying type $s$ of lane markings. Neural networks have also been used to attempt to detect lanes and control vehicles [3], but have difficulties on roads not included in their training set. Techniques using tangent vectors have also been shown to be quite robust on well-marked roads, but can fail when lane markings are not well defined [10].

Others have attempted to overcome problems of differing lane markings by using multiple detectors. For example, Gehrig Et. Al. [4] detect bots dots on California highways using a specific detector for bots dots using matched filters and detect solid lane markings using more classical methods.

Others, such as Southall Et. Al. [5], propose stochastic methods have also been proposed to overcome lighting and road changes while Broggi Et. Al. [6] developed the GOLD system for robust obstacle and lane detection. 
Studies have been done in the state of the art of lane detection [7]. While these methods are all very effective at solving these problems, they tend too be very specific to particular road types or conditions. Robust lane detection remains an unsolved problem because in order to have a robust lane detector, the system must be invariant to all manners of road markings, road conditions, lighting changes, shadowing, and occlusion.

\section{Steerable Filters for Lane Detection}

Steerable filters have a number of desirable properties that make them excellent for a lane detection application. First, they can be created to be separable in order to speed processing. By separating the filters into an $\mathrm{X}$ and $\mathrm{Y}$ component, the convolution of the filter with an image can be split into two convolutions using the $X$ components and $\mathrm{Y}$ components separately. Second, a finite number of rotation angles for a specific steerable filter are needed to form a basis set of all angles of that steerable filter. This allows us to see the response of a filter at a given angle and therefore to tune the filter to specific lane angles or look at all angles at once.

\subsection{Formulation of the steerable filters}

The steerable filters used for the bots dot and lane detection are based on second derivatives of twodimensional Gaussians. Figure 2 shows an example of a steerable filter basis set constructed from equations 1,2 , and 3.

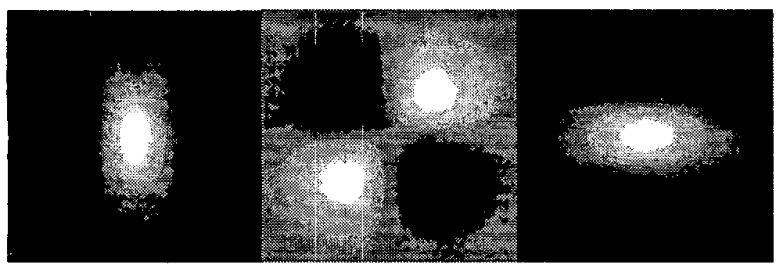

Figure $2 \mathrm{~A}$ basis set for steerable filters based on the second derivatives of a two-dimensional Gaussian

$$
\begin{aligned}
& G_{x x}(x, y)=\frac{\partial^{2}}{d x^{2}} e^{\frac{-\left(x^{2}+y^{2}\right)}{\sigma^{2}}} \\
& =-\left(\frac{2 x^{2}}{\sigma^{2}}-1\right) \frac{2}{\sigma^{2}} e^{\frac{-x^{2}}{\sigma^{2}}} e^{\frac{-y^{2}}{\sigma^{2}}}
\end{aligned}
$$

$$
\begin{aligned}
& G_{x y}(x, y)=\frac{\partial}{d x} \frac{\partial}{d y} e^{\frac{-\left(x^{2}+y^{2}\right)}{\sigma^{2}}} \\
& =\frac{2 x}{\sigma^{2}} e^{\frac{-x^{2}}{\sigma^{2}}} \frac{2 y}{\sigma^{2}} e^{\frac{-y^{2}}{\sigma^{2}}} \\
& G_{y y}(x, y)=\frac{\partial^{2}}{d y^{2}} e^{\frac{-\left(x^{2}+y^{2}\right)}{\sigma^{2}}} \\
& =-\left(\frac{2 y^{2}}{\sigma^{2}}-1\right) \frac{2}{\sigma^{2}} e^{\frac{-x^{2}}{\sigma^{2}}} e^{\frac{-y^{2}}{\sigma^{2}}}
\end{aligned}
$$

It has been shown that the response of any rotation of the $\mathrm{G}_{\mathrm{xx}}$ filter can be computed using the equation 4 [1].

$$
\begin{aligned}
& G 2^{\theta}(x, y)=G_{x x} \cos (\theta)^{2}+G_{y y} \sin (\theta)^{2} \\
& -2 G_{x y} \cos (\theta) \sin (\theta)
\end{aligned}
$$

Taking the derivative of (4), setting it equal to 0 , and solving for $\theta$, we can find the values of $\theta$ that correspond to the minimum and maximum responses. These responses can be computed by the formulas given in 5 and 6.

$$
\begin{gathered}
\theta_{\min }=\tan ^{-1}\left(\frac{\mathrm{G}_{\mathrm{xx}}-\mathrm{G}_{\mathrm{yy}}-\mathrm{A}}{2 \mathrm{G}_{\mathrm{xy}}}\right) \\
\theta_{\mathrm{max}}=\tan ^{-1}\left(\frac{\mathrm{G}_{\mathrm{xx}}-\mathrm{G}_{\mathrm{yy}}+A}{2 \mathrm{G}_{\mathrm{xy}}}\right) \\
A=\sqrt{\mathrm{G}_{\mathrm{xx}}{ }^{2}-2 \mathrm{G}_{\mathrm{xx}} \mathrm{G}_{\mathrm{yy}}+\mathrm{G}_{\mathrm{yy}}{ }^{2}+4 \mathrm{G}_{\mathrm{xy}}{ }^{2}}
\end{gathered}
$$

\subsection{Application of steerable filters to road marking detection}

Using the formulas 4,5 , and 6 , we can find the values and angles of the minimum and maximum responses, or the response at a given angle. This is useful for detecting bots dots because, for circular objects, the minimum and maximum responses will be very similar. For detecting lanes, the response in the direction of the lane should be near the maximum, and the minimum response should be low.

By applying a threshold to the minimum filter response we can find the bots dots within an image. Also, applying a threshold to the difference between the response in the direction of the lane marking and the minimum response, 
we can detect lanes of a specific angle. Figure 3 shows a typical California highway scene with lane markings consisting of both Bots Dots and lines. Figure 4 shows the image after being filtered and thresholded by the minimum value. Figure 5 shows the response to lines in the orientation of the current lane parameters.

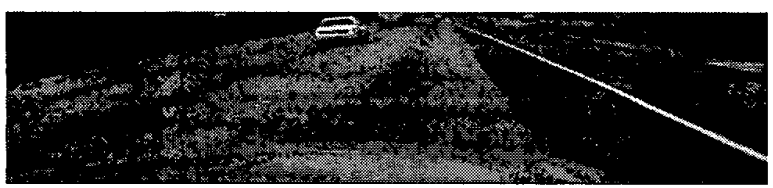

Figure $3 \mathrm{~A}$ typical highway scene in California.

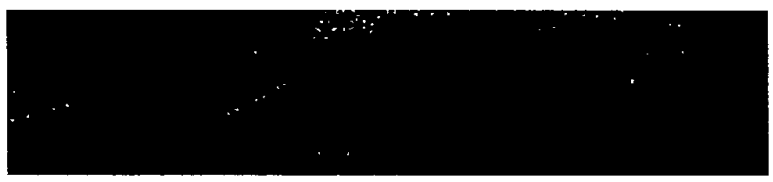

Figure 4 Results of filtering for Bots Dots.

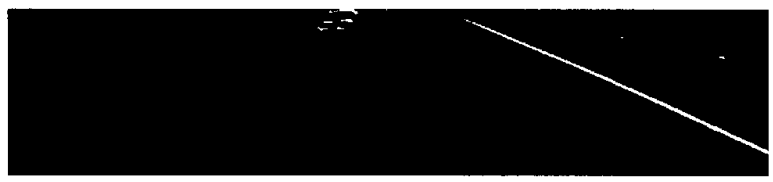

Figure 5 Results from filter for a line tuned to the lane angle.

These results show the usefulness of the steerable filter set for relatively normal highway conditions. This filtering technique is also very useful for dealing with shadowed regions of road. Figure 6 below shows a road section that is shadowed by trees and the filter response for the lane when it is tuned for that lane angle.

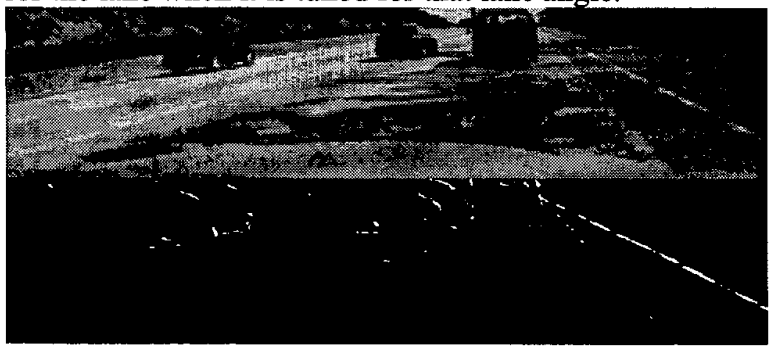

Figure 6 Filter results when lane markings are shadowed with complex shadows and non-uniform road materials.

\section{Lane Detection System}

The overall system that we have implemented is diagramed in Figure 7. The video input to the system are taken from a forward looking rectilinear camera for our test results, but can be taken from any number of cameras on our test bed vehicle. For more information on this test bed, please refer to [11]. Information about the vehicles state including wheel velocities and steering angle are acquired from the car via the internal CAN bus. These inputs are then fed into the tracking system to determine the state of the vehicle and road. The lane angles in the image coordinates are then feed back into the filtering algorithm in order to tune the filters for specific lanes.

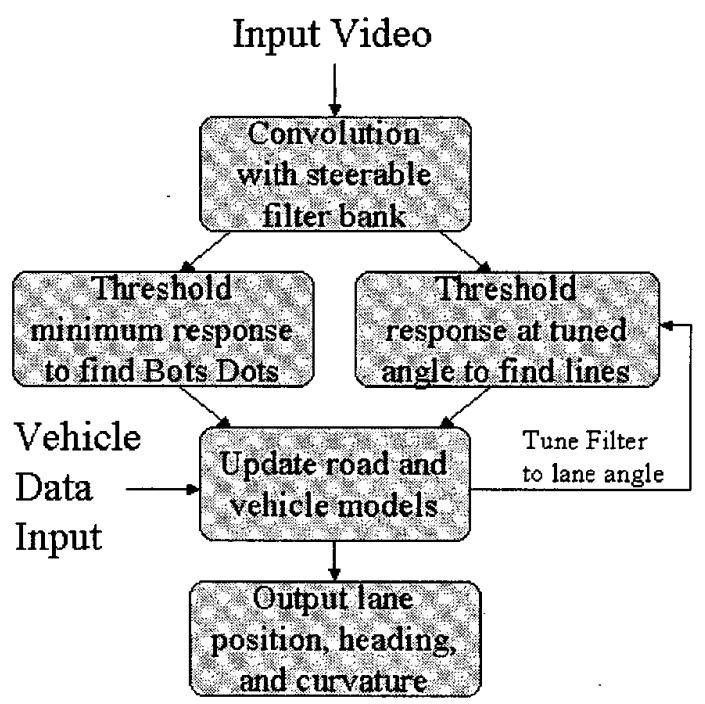

Figure 7 The Lane Tracking System Flow Chart.

\subsection{Road Modeling}

The road model used in our system is similar in form to that used in [5]. The state variable include the vehicle offset from the center of the lane, the vehicle heading with respect to the lane, the rate of change of the lane heading, the steering angle of the vehicle, and the velocity of the vehicle. Currently lane curvature is estimated using the steering angle and the rate of change of the lane heading and is only estimated at the vehicles location. The camera pitch and lane width are assumed constant in this implementation. The vehicle state is updated in time each frame as well as updated from the measurements via a Kalman filter, which is described at the end of the next section.

\subsection{Lane Tracking}

In order to perform robust tracking, some more postprocessing on the filter results is performed. First, only the filter candidates within the vicinity of the lanes are used in updating the lanes. This removes outliers from other vehicles and extraneous road markings. Secondly, for each lane, the first and second moments of the point candidates are computed. Straight lane markings should be aligned to that there is a high variance in the lane heading direction and a low variance in the other direction. Outliers are then removed based on these statistics. Because the algorithm uses a local search about 
the lanes for candidates, it requires initialization. In testing, it was sufficient to initialize the lane tracker position and trajectory to zero (corresponding to the center of the lane).

These computed headings and positions in image space are then transformed into real-world coordinates via an inverse perspective calculation. The state variables are then updated using these measurements as well as measurements of steering angle and wheel velocity provided by the vehicles CAN bus.

These measurements are then feed into a discrete time Kalman filter for the road and vehicle state as described in section 3.1. The system and measurement equations as well as the Kalman update equations at time $\mathbf{k}$ are shown below.

$$
\begin{gathered}
x_{k+1 \mid k}=A x_{k \mid k}+B u_{k} \\
y_{k}=C x_{k} \\
P_{k+1 \mid k}=A P_{k} A^{T}+Q \\
P_{k+1 \mid k+1}^{-1}=C^{T} R^{-1} C+P_{k+1 \mid k}^{-1} \\
K=P_{k+1 \mid k+1} C^{T} R^{-1} \\
x_{k+1 \mid k+1}=x_{k+1 \mid k}+K\left(y_{k+1}-C x_{k+1 \mid k}\right)
\end{gathered}
$$

\section{Experimental Results and Evaluation}

Testing was performed on southern California highways. These highways contained overpasses, both Bots Dots lane markers and painted line lane markers, shadowing from trees and vehicles, and changes in road surface material. Below are images taken from these tests that demonstrate the system working under these conditions. The lines to the side of the vehicles are the detected lanes, the line emanating from the center of the vehicle is the vehicles trajectory based on internal sensors, and the horizontal line is the distance to the lead vehicle, which is obtained from a LASER RADAR range finder built into the vehicle.

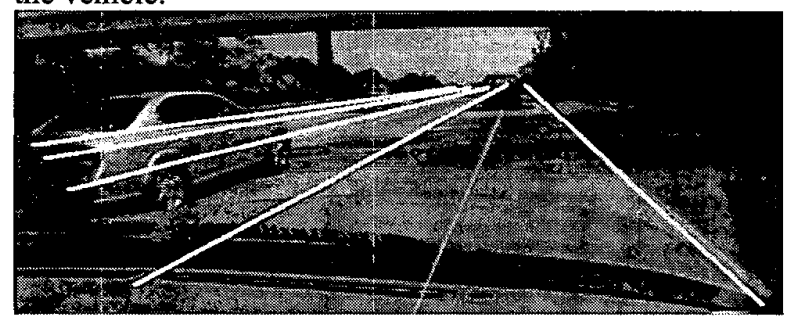

Figure 8 Results with clutter from nearby cars and tree shadows. (Frame 13 of the test sequence)

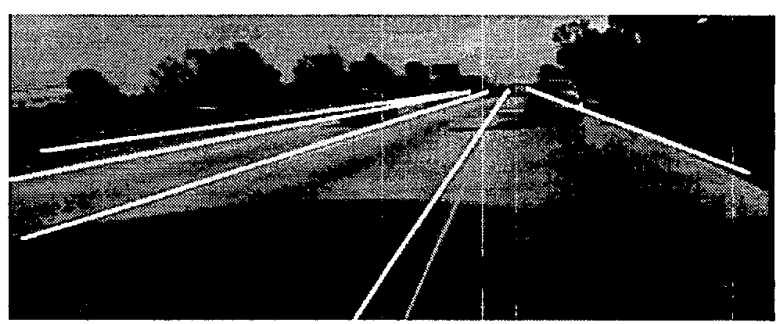

Figure 9 Results during a lane change. (Frame 258 of the test sequence)

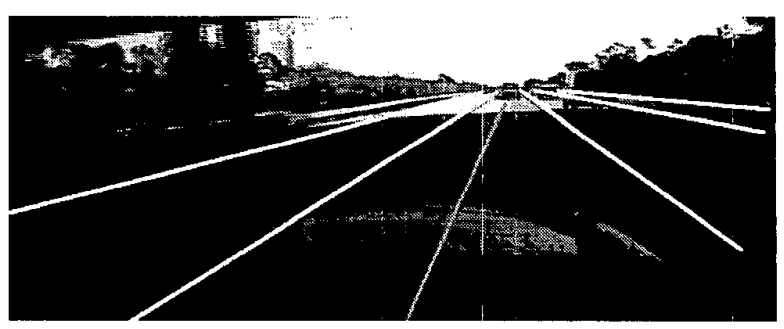

Figure 10 Results with lighting changes from going through an overpass. (Frame 1657 of the test sequence)

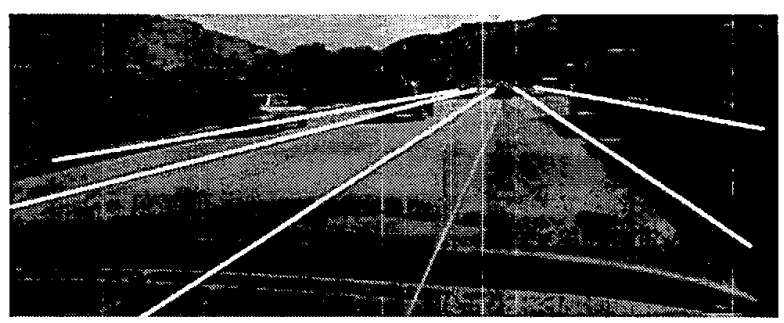

Figure 11 Results with shadowing from trucks and only Bots Dots lane markings. (Frame 4043 of the test sequence)

The following graphs (Figures 12 and 13) show the lane tracking and vehicle state information extracted from a run shown in figures 8-11 above.

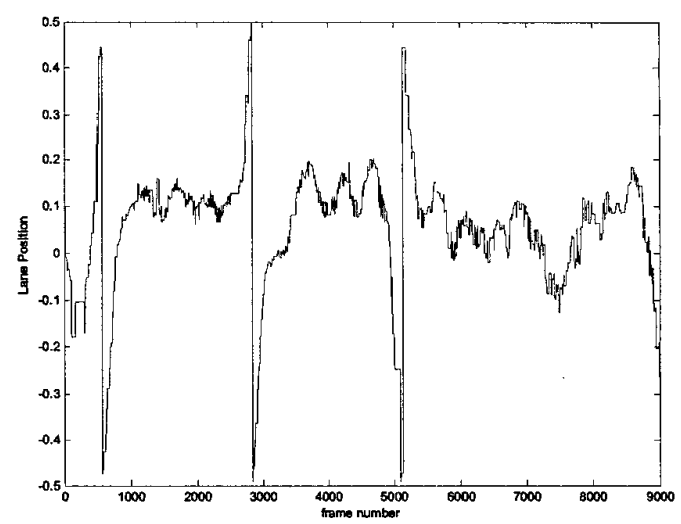

Figure 12 Lane Position Output from the lane Tracking System 


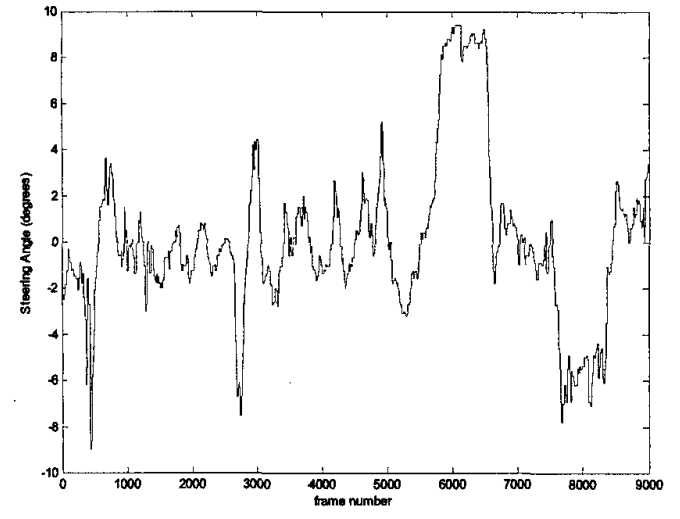

Figure 13 Steering angle output from the lane tracking system

Lane changes occur around frames 500, 2800, and 5200 and are successfully detected by the system. The road is straight in frames 0 though 3700 . After that the road curves as can be seen in the sustained steering angles.

\section{Summary and Conclusions}

Lane detection is often complicated by varying road markings, clutter from other vehicles and complex shadows, lighting changes from overpasses, occlusion from vehicles, and varying road conditions. In this paper we have presented a solution to the lane detection problem that shows robustness to these conditions. We have shown that using a steerable filter bank provides. robustness to lighting changes, road marking variation, and shadowing. Further post-processing based on the statistics of the road marking candidates increases the robustness to occlusion by other vehicles and changing road conditions. Future work will include expanding the road model to incorporate piecewise estimates of trajectory and curvature. Also, this system can also be combined into a vehicle surround analysis system such as that described in Huang et. al.[9] to create a intelligent vehicle driver assistance system.

\section{Acknowledgments}

The authors of this paper would like to thank UC Discovery Grant (Digital Media Innovations program), Nissan Motor Co. LTD., and their colleagues at the Computer Vision and Robotic Research Laboratory, especially Dr. Tarak Gandhi.

\section{References}

[1] W. T. Freeman and E. H. Adelson. "The design and use of steerable filters", PAMI, 13(9):891--906, 1991.

[2] J. McDonald. "Detecting and tracking road markings using the Hough transform." Proc. of the Irish Machine Vision and Image Processing Conference, 2001.

[3] D. Pomerleau. Ralph: "Rapidly adapting lateral position handler." Proc. IEEE Symposium on Intelligent Vehicles, September 25-26, 1995.

[4] Stefan Gehrig, Axel Gern, Stefan Heinrich and Bernd Woltermann, Lane recognition on poorly structured roads - The Bot Dot problem in California", Proc. of the $5^{\text {th }}$ Conference on Intelligent Transportation Systems, 2002.

[5] J. B. Southall and C.J. Taylor "Stochastic road shape estimation" International Conference on Computer Vision ,pp. 205-212, June 2001

[6] M. Bertozzi and A. Broggi. "GOLD: a Parallel Real-Time Stereo Vision System for Generic Obstacle and Lane Detection." IEEE Transactions on Image Processing, 1997.

[7] J. Kosecka, R. Blasi, C. Taylor and J. Malik. "A Comparative Study of Vision-Based Lateral Control Strategies for Autonomous Highway Driving". In IEEE Int. Conf. on Robotics and Automation, pp 1903-1908, May 1998.

[8] C.J. Taylor and Jitendra Malik and Joseph Weber. "A Real-Time Approach to Stereopsis and Lane-Finding." Intelligent Vehicles 1996, pp 207-212

[9] K. Huang, M. M. Trivedi, T. Gandhi, "Driver's View and Vehicle Surround Estimation using Omnidirectional Video Stream," Proc. IEEE Intelligent Vehicles Symposium, Columbus, OH, pp. 444-449, June 9-11, 2003

[10] H. Furusho, R Shirato, M. Shimakage. "A Lane Recognition Method Using the Tangent Vectors of White Lane Markers," 6th International Symposium on Advanced Vehicle Control, Sept. 9-13, 2002.

[11] J. McCall, O. Achler, M. M. Trivedi. "The LISA-Q Human-Centered Intelligent Vehicle Test Bed" To Appear in Proc. IEEE Intelligent Vehicles Symposium, Parma, Italy, June 14-17, 2004. 